Classification

Physics Abstracts

$06.50-42.30$

\title{
Automated segmentation algorithm for electron microscope images with strong grey level fluctuations
}

\author{
Xin Zheng and Max Kolb \\ Laboratoire de Chimie Théorique, Ecole Normale Supérieure de Lyon, 46 Allée d'Italie, 69364 Lyon \\ cedex 07, France and Institut de Recherches sur la Catalyse, CNRS, 2 Ave. A. Einstein, 69626 \\ Villeurbanne cedex, France
}

(Received January 25; accepted June 10, 1994)

\begin{abstract}
An automated algorithm for image segmentation based on nucleation and growth of the background is presented. The graytone parameters of the algorithm are chosen by the algorithm itself. Only a single graytone cutoff remains to be chosen by hand. This cutoff is more general than the graytone parameters themselves, as it can be fixed for a whole class of images. Different ways to implement the automated choice of the graytone parameters are investigated. The role of the geometrical parameters of the automated algorithm is also discussed.
\end{abstract}

\section{Introduction.}

People often use electron microscopy to study the sintering of supported catalytic metal particles [1]. This study necessitates the identification of the particles to be analysed from their electron micrograph, thus we have proposed an image segmentation alogorithm based on the nucleation and growth of the background [2]. This method consists of three steps. One first detects the background nuclei; then the nuclei grow - simultaneously and iteratively - into the background, based on the criterion of discontinuity of neighbouring regions. Finally, the boundaries of the already segmented regions are refined by comparing the graytone difference between boundary and background with the graytone difference between boundary and object. The similar approach has been proposed by Adams [3].

Image segmentation algorithms used to extract geometrical objects from graytone images always depend on geometrical parameters and/or graytone parameters, these two kinds of parameters are respectively related to geometrical features and to the graytone information. The three basic types of image segmentation algorithms - thresholding, edge detection and region extraction [4] - all depend on parameters which have to be adjusted for each image. For example, the image segmentation method of global thresholding [5] uses a threshold value to isolate objects from the background of an image. For image segmentations depending on edge detection [6] one must choose a threshold and a mask to search for the edge points; the size of the mask approximately corresponds to the thickness of the object boundary. The region extraction method [7] is based 
on the similarity of the image characteristics of the subdivisions of an image; the method needs an initial subdivision and a criterion of similarity.

In many cases, the geometrical parameters are chosen by hand for each image because of the following considerations: in a graytone image, the geometrical characteristics of the objects are unknown to the computerised image processing system until the objects are extracted by the segmentation operations. The geometrical parameters only relate to the geometry of the objects, therefore they do not change from image to image. By contrast, the graytone characteristics are dependent on the external conditions (such as illumination), they do change with varying conditions. The attention of most segmentation algorithms therefore concentrates on how to automatically choose the values of these graytone parameters directly by the image segmentation algorithm [8].

In its simplest form, the nucleation and growth method requires five parameters: three of them are geometrical parameters, the others two are graytone parameters. So far, the two graytone parameters of this algorithm have been chosen by hand. We call as the basic algorithm the simplest form of our segmentation method.

In the present study, we first briefly review our basic image segmentation algorithm based on nucleation and growth of the background. Then, a modified image segmentation algorithm with automatic choice of the graytone parameters is developed for a fixed set of the geometrical parameters. Finally, we study the effect of different choices of the geometrical parameters using the new algorithm. In our study, the graytone and the geometric parameters are investigated separately because the effects of the five parameters are strongly correlated.

\section{Basic image segmentation algorithm by nucleation and growth.}

Images are represented on a square network of points, the points of the network are called pixels. The lattice constant of the network is equal to one. The position of each pixel is given by $(x, y)$ : $x$ represents the row coordinate and $y$ the column coordinate. The brightness of each pixel is digitized into integer grey levels from 0 (white) to 255 (black). The grey level of the pixel located at $(x, y)$ is written as $f(x, y)$.

For a square network two definitions of adjacency between any two neighboring pixels are used: four-adjacency and eight-adjacency. If a pixel is one of the four nearest neighbors of another pixel, the two pixels are four-adjacent. If a pixel is one of the eight nearest or next nearest neighbors, the two pixels are eight-adjacent.

The description of our algorithm is done using the concept of sets. The set of all pixels in a given image is referred to as $F$. The set $F$ can be divided into two subset: the objects $O$ (to be segmented) and the background $B$. The intersection of $B$ and $O$ is empty, and the union of the two subsets is exactly equal to $\mathrm{F}$.

A pixel $(x, y)$ is called 4-adjacent to a non-empty set $\mathrm{X}$ if it is not included in $\mathrm{X}$ but at least one of its four-adjacent neighbors is included in $X$. For a non-null subset $X$ of $F$ with $X \neq F$, there is a non empty accompanying subset $\partial_{4} \mathrm{X}$ (the internal boundary with respect to $\mathrm{F}$ ), which is composed of the pixels satisfying the following conditions:

1) $(x, y) \in \mathbf{X}$,

2) $(x, y)$ is four-adjacent to $F-X$. 
For each pixel at $\left(x_{0}, y_{0}\right)$, we can define a square neighborhood $\mathrm{D}\left[\left(x_{0}, y_{0}\right) ; L\right]$, composed of the pixels in a neighborhood centered at $\left(x_{0}, y_{0}\right)$ :

$$
\mathrm{D}=\left\{(x, y) ;(x, y) \in \mathrm{F} \text { and }\left|x-x_{0}\right| \leq L / 2 \text { and }\left|y-y_{0}\right| \leq L / 2\right\}
$$

where $L$ is the side lengths of the square. Clearly $\mathrm{D}$ is a subset of $\mathrm{F}$.

For a non-null set $\mathrm{X}$ with $N$ elements (pixels), we introduce $A[\mathrm{X}]$, the average grey level of the pixels in the set $\mathrm{X}$ :

$$
A[\mathrm{X}]=\sum_{(x, y) \in \mathbf{X}} f(x, y) / N
$$

The three steps of the nucleation and growth method use three functions $I, J$ and $K$, which are defined on $\mathrm{F}$ or on subsets of it and which depend on $f(x, y)$. The three steps are described in the following.

2.1 NUCLEATION OF THE BACKGROUND. - In order to detect the background nuclei, we define an operation which maps the original grey level function $f(x, y)$ into a new function $I\left(x, y ; L_{\mathrm{I} 1}, L_{\mathrm{I} 2}\right)$ :

$$
I=A\left[\mathrm{D}_{1}\right]-A\left[\mathrm{D}_{2}-\mathrm{D}_{1}\right]
$$

where $\mathrm{D}_{1}=\mathrm{D}\left[(x, y) ; L_{\mathrm{I} 1}\right]$ and $\mathrm{D}_{2}=\mathrm{D}\left[(x, y) ; L_{\mathrm{I} 2}\right]$, with $L_{\mathrm{I} 2}>L_{\mathrm{I} 1}$. The function $I$ determines the difference of the grey levels between two regions: $D_{1}$ and $D_{2}-D_{1}$ (pixels in $D_{2}$ but not in $D_{1}$ ). The latter surrounds the former.

In the first step, $I(x, y)$ is computed for every point of the image. The initial background set $\mathrm{B}$ is defined as the set of all pixels $(x, y)$ for which $I<M_{I}$, where $M_{I}$ is a (negative) graytone parameter. Correspondingly, $\mathrm{O}=\mathrm{F}-\mathrm{B}$ is the initial object set. The initial B consists of many disjoint regions which we call the background nuclei.

2.2 INVASION OF THE BACKGROUND. - In order to control the growth of the nuclei using the property of discontinuity, we define a new function $J\left(x, y ; L_{\mathrm{I} 1}, L_{\mathrm{J} 2}\right)$ for all pixel $(x, y)$ satisfying the following two conditions:

(1) $(x, y) \in \partial_{4} \mathrm{O}$

(2) $\left(D_{2}-D_{1}\right) \cap B$ is not empty.

$\mathrm{D}_{1}$ and $\mathrm{D}_{2}$ are different neighborhoods of the pixels $(x, y): \mathrm{D}_{1}=\mathrm{D}\left[(x, y) ; L_{\mathrm{I} 1}\right], \mathrm{D}_{2}=\mathrm{D}[(x, y)$; $L_{J 2}$ ], with $L_{J 2}>L_{I 1} . J\left(x, y ; L_{I 1}, L_{J 2}\right)$ then is defined by:

$$
J=A\left[\mathrm{D}_{1}\right]-A\left[\left(\mathrm{D}_{2}-\mathrm{D}_{1}\right) \cap \mathrm{B}\right]
$$

A new background set is now defined as the union of the old background set and those pixels for which $J$ is defined and satisfies $J<M_{J}$, where $M_{J}$ is a second (positive) graytone parameter. Then the new object set is the complement of the new background set, $\mathrm{O}=\mathrm{F}-\mathrm{B}$.

The above operation is repeated with the same value $M_{J}$ until the segmentation does not change any more. 
2.3 REFINEMENT OF THE BOUNDARY. - The function $K\left(x, y ; L_{I 1}, L_{J 2}\right)$, in conjunction with $J$, is designed to refine the boundaries of the object regions that form the set $\mathrm{O}$ obtained in the previous step. $K\left(x, y ; L_{I 1}, L_{J 2}\right)$ is defined by

$$
K=A\left[\mathrm{D}_{1}\right]-A\left[\left(\mathrm{D}_{2}-\mathrm{D}_{1}\right) \cap \mathrm{O}\right]
$$

for all pixels satisfying the following three conditions:

(1) $(x, y) \in \partial_{4} \mathrm{O}$,

(2) $\left(\mathrm{D}_{2}-\mathrm{D}_{1}\right) \cap \mathrm{O}$ is not empty,

(3) $\left(D_{2}-D_{1}\right) \cap B$ is not empty.

$\mathrm{D}_{1}$ and $\mathrm{D}_{2}$ again are different rectangle neighborhoods: $\mathrm{D}_{1}=\mathrm{D}\left[(x, y) ; L_{I 1}\right]$ and $\mathrm{D}_{2}=\mathrm{D}[(x, y)$; $\left.L_{J 2}\right]$.

Both $K\left(x, y ; L_{I 1}, L_{J 2}\right)$ and $J\left(x, y ; L_{I 1}, L_{J 2}\right)$ are evaluated for every pixel $(x, y)$ satisfying the above three conditions. A new object set $\mathrm{O}$ is then obtained from the old one by removing the pixels for which $|K(x, y)|>|J(x, y)|$. The new background set is the complement of the new object set, $\mathrm{B}=\mathrm{F}-\mathrm{O}$. The operation is also iterated until $\mathrm{B}$ does not change any more. Note that this step does not require any graytone parameters.

\section{Automated image segmentation algorithm based on nucleation and growth.}

First, we propose two ways to calculate $M_{I}$ from the original graytone image, and three possible choices for fixing $M_{J}$ in the original image and from the initial segmented image obtained from the first segmentation step. We call this operation the automated parameter choice. The selection of the best method remains to be decided empirically for each type of image.

Next, we add an extra step to the basic segmentation operations defined above in order to improve the segmentations with the automated parameter choice. The reason is that the automated parameter choice for a whole series of images is not expected to perform as well as the best manual choice for each individual image. The extra step is the cleaning of the segmented images: the removal of internal holes in the object regions and the removal of the spurious object regions.

To illustrate the automatic choices of the graytone parameters and the automated image segmentation algorithm, we use a group of test images with varying contrast (Figs. 1-6). The elementary scale is set such that each image contains particles with the same typical sizes in the range between 15 and 30 pixels. Correspondingly, we can fix the same geometrical parameters for all the images: $L_{I 1}=5, L_{I 2}=11$ and $L_{J 2}=41$.

3.1 AutOMATIC CHOICE OF THE PARAMETER $M_{I}$. - As a first automatic choice for $M_{I}$, which we call $M_{I 1}$, we calculate the average of $I(x, y)$ over all pixels for which $I(x, y)<0$ :

$$
M_{I 1}=\frac{\sum_{(x, y), I(x, y)<0} I(x, y)}{\sum_{(x, y), I(x, y)<0} 1}
$$

A second (lower) threshold value for $M_{I}$, which we call $M_{I 2}$, can be obtained by calculating the average of $I(x, y)$ over all pixels for which $I(x, y)<M_{I 1}$. 
Empirically, for the transmission electron micrographs that we encounter in catalysis, the second choice of $M_{I}$ turns out to be better than the first one. The reason is that for $M_{I 2}$ fewer spurious background nuclei appear in the first step of our segmentation algorithm (the detection of background nuclei). In particular, fewer spurious nuclei overlap with or lie within the true object regions. Therefore, we will always use the value $M_{I 2}$ for the graytone parameter $M_{I}$ in the automated image segmentation algorithm.

3.2 AUtOMATIC CHOICE OF THE PARAMETER $M_{J}$. - The first way to fix $M_{J}$ calculates $J(x, y)$ for each pixel which satisfies:

1) $(x, y) \in \mathrm{O}$,

2) $\left(D_{2}-D_{1}\right) \cap B$ is not empty,

where $\mathrm{O}$ and $\mathrm{B}$ is the initial segmentation as defined in the first step. $M_{J_{1}}$ then is defined as the average of $J(x, y)$ over those pixels for which $J(x, y)$ is defined and positive:

$$
M_{J 1}=\frac{\sum_{(x, y), J(x, y)>0} J(x, y)}{\sum_{(x, y), J(x, y)>0} 1}
$$

A second choice, $M_{J 2}$, is obtained by restricting the average in equation (7) to the pixels belonging to the boundary $\partial_{4} \mathrm{O}$ instead of the entire set $\mathrm{O}$ :

1) $(x, y) \in \partial_{4} \mathrm{O}$,

2) $\left(D_{2}-D_{1}\right) \cap B$ is not empty.

A third value for $M_{J}$ (larger than $M_{J 2}$ ), $M_{J 3}$, is obtained by repeating the second operation twice: first, we calculate $M_{J 2}$ as above; then we calculate the restricted average over all the pixels for which $J(x, y)>M_{J 2}$. This yields $M_{J 3}$.

Let us illustrate the three choices of $M_{J}$ in two images (Figs. 1-2). Figure 1 shows an artificial image and its segmentations for $\left(M_{I}, M_{J}\right)$ equal to $\left(M_{I 2}, M_{J 1}\right),\left(M_{I 2}, M_{J 2}\right)$ and $\left(M_{I 2}, M_{J 3}\right)$, respectively. The original image (Fig. 1a) consists of two particles of different sizes and with different grey levels in a noisy environment. The background of the original image is non-uniform.

We applied the same methods to a portion of an electron micrograph containing a few metal particles on an amorphous support (Fig. 2). The boundary of the largest particle in figure $2 \mathrm{a}$ is very irregular.

Comparing the segmentations, one concludes that the second and third methods are better for the image of figure 2a: the particles extracted coincide closely with the ones in the original image and very few spurious regions are extracted by mistake.

Even with the choices $M_{J 2}$ and $M_{J 3}$ for $M_{J}$, there are two problems that need to be solved (see for example Fig. 1):

1) there are small holes inside of the object regions,

2) a few unwanted object regions are present.

These difficulties motivate us to include a cleaning operation in the next section.

The two examples illustrate that the segmentation results are quite sensitive to the choice of $M_{J}$. For all three choices of $M_{J}, M_{J}=M_{J 1}, M_{J 2}, M_{J 3}$, unwanted regions appear (see Fig. 1). In addition, for $M_{J}=M_{J 1}$ object regions are invaded by the background nuclei (see Fig. 2). In 


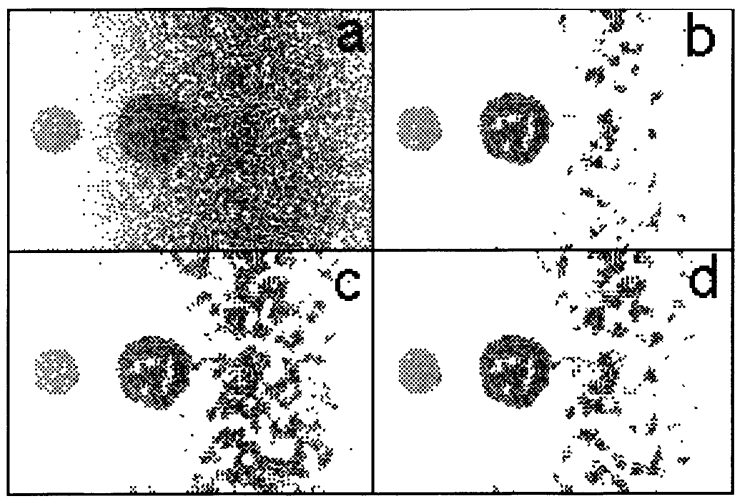

Fig. 1. - Segmentations of an artificial image using different methods to choose $M_{J}\left(L_{I 1}=5, L_{I 2}=\right.$ $\left.11, L_{J 2}=41\right)$, a) original image $\left(L_{x}=100, L_{y}=150\right)$, b) the first method $\left(M_{I 2}, M_{J 1}\right)$, c) the second method $\left(M_{I 2}, M_{J 2}\right)$, d) the third method $\left(M_{I 2}, M_{J 3}\right)$.

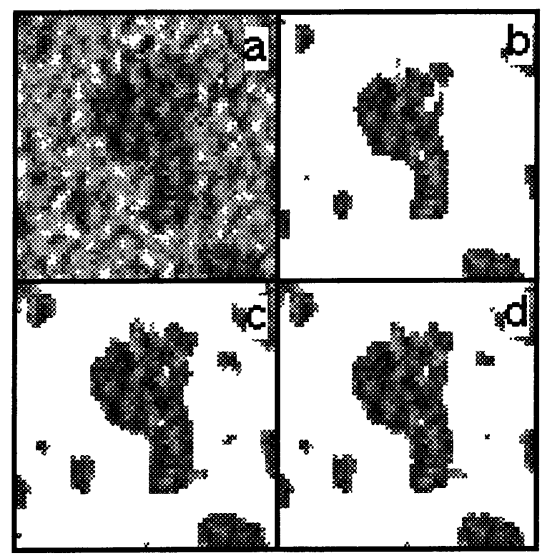

Fig. 2. - Segmentations of a small portion of transmission electron micrograph containing a few metal particles [1] using different methods to choose $M_{J}\left(L_{I 1}=5, L_{I 2}=11, L_{J 2}=41\right)$, a) original image $\left.\left(L_{x}=56, L_{y}=55\right), \mathrm{b}\right)$ the first method $\left.\left(M_{I 2}, M_{J 1}\right), \mathrm{c}\right)$ the second method $\left(M_{I 2}, M_{J 2}\right)$, d) the third method $\left(M_{I 2}, M_{J 3}\right)$.

general, when $M_{J}$ is large, the background nuclei can grow into object regions and the objects are lost or distorted. The opposite happens when $M_{J}$ is small: the background nuclei do not invade the entire background and many unwanted object regions are present. Our results show that it is difficult to avoid both problems. Therefore, we use a small $M_{J}$ such as $M_{J 2}$ (in our examples both $M_{J 1}$ and $M_{J 3}$ happen to be larger than $M_{J 2}$ ) and add another segmentation step to remove the unwanted regions. We now define this step and show that $M_{J}=M_{J 2}$ is a good choice for the cleaning operation. 
3.3 Cleaning of THE IMAGE. - The starting point for the cleaning is the previously obtained segmented image, i.e. $\mathrm{O}$ and $\mathrm{B}$, by the basic algorithm, using $M_{I}=M_{I 2}$ and $M_{J}=M_{J 2}$. The cleaning of the segmented images consists of two operations: filling of internal holes and removal of unwanted regions. We explain the two operations in the following.

We first remove the internal holes from the objects. The internal holes of the object regions are filled using the standard procedure of checking whether they are connected to the boundary of the image [9]. Having filled these holes, we obtain a new object set $O_{1}$ and a new background set $\mathrm{B}_{1}=\mathrm{F}-\mathrm{O}_{1}$.

We now remove the unwanted regions by an iteration process. Each iteration step consists of applying the segmentation steps 2) and 3) of the basic nucleation and growth method as defined above (Eqs. (3-5)). In contrast with the segmentation algorithm defined above the value of $M_{J}$ now changes at each iteration. The iteration procedure terminates when the relative change in $M_{J 2}$ from one iteration to the next is less than a predetermined value.

The iteration procedure is defined as follows: Start from the segmented image, $\mathrm{O}_{1}$ and $\mathrm{B}_{1}$. At the iteration step $i\left(i>1\right.$ ), we fix $M_{J}$ at $M_{J 2, i-1}$ (the value $M_{J 2}$ evaluated from $f(x, y)$, and the previous segmentation $\mathrm{O}_{i-1}$ and $\mathrm{B}_{i-1}$ ). The geometric parameters $L_{I 1}$ and $L_{J 2}$ are unchanged. Applying the second and third segmentation steps, using $M_{J 2, i-1}$, generates the new segmented image: $\mathrm{O}_{i}$ and $\mathrm{B}_{i}$. The operation of removing unwanted regions stops when the rate of change of $M_{J 2}$ falls below a given value. We measure the rate of change by the variable $p_{i}$, which is defined by

$$
p_{i}=\frac{M_{J 2, i}-M_{J 2, i-1}}{M_{J 2, i-1}}
$$

When $p_{i}<p_{\mathrm{c}}$, where $p_{\mathrm{c}}$ sets the limit for the relative change of $M_{J 2}$, the iteration stops and $\mathrm{O}_{i-1}$ and $\mathrm{B}_{i-1}$ are taken to be the final segmented image.

Using the cutoff $p_{\mathrm{c}}$ to decide when to stop the cleaning operations avoids to control the iteration process manually. Furthermore, different images can be treated simultaneously. This is close to our final goal of finding segmentations that are automatic and unbiased for entire series of images.

3.4 AUTOMATED IMAGE SEGMENTATION ALGORITHM. - After having presented the automated parameter choice and the cleaning operation, we can now summarize our automated image segmentation algorithm. The algorithm consists of six steps: 1) determining $M_{I 2}$ as the graytone parameters $M_{I}, 2$ ) nucleation of the background (the first step in the basic algorithm), 3) calculating $M_{J}=M_{J 2}$ to be used in the second step of the basic algorithm, 4) growing the background, 5) refining the boundaries of the extracted regions, and 6) iterative cleaning of the segmented image. The present automated algorithm only has a single graytone cutoff to be selected by hand.

3.5 APPLiCATIONS. - The difficulty to systematically analyse series of images stems from the different contrast conditions which require the manual choice of the graytone parameter values for each image. In the present automated procedure, the manual choice of the two graytone parameters is replaced by a single cutoff. For a series of images with changing contrasts, it is possible to find a generally valid cutoff and obtain good results for the segmentation. This is illustrated in the following.

We empirically set $p_{c}=0.23$ for the segmentations of all the images. The results for a variety of images are presented in figures 3-6. The geometrical parameters are fixed at $L_{I 1}=5, L_{I 2}=11$ and $L_{J 2}=41$, as discussed above.

As a first example we show the entire segmentation process including the cleaning operation for the examples already presented in figures 1 and 2 . The final segmentation of figure $1 \mathrm{a}$ by use of the automated method is shown in figure 3a. It is better than the previous method without the cleaning 
step (in Fig. 1). A few unwanted regions still remain, because their gray levels are much higher than the gray levels of the local backgrounds surrounding them. The final segmentation of figure $2 \mathrm{a}$ by use of the automated method is presented in figure $3 \mathrm{~b}$. In this case the final segmentations with the automated parameter choice and without the cleaning step work equally well.
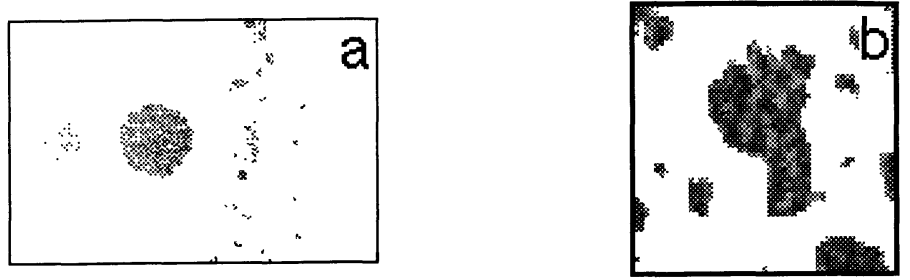

Fig. 3. - Automatic segmentations of images including the final cleaning step with $L_{I 1}=5, L_{I 2}=11, L_{J 2}=$ 41 , a) segmented image from figure $1 a, b)$ segmented image from figure $2 a$.

Next we consider several images from catalysis. Figure 4a and figure 5a are, respectively, small portions of two different electron micrographs of metallic particles. Their contrast is much weaker in comparison with the first two examples. Figure 4 contains a few particles, whereas figure 5 contains many particles. The segmentations of figure $4 \mathrm{a}$ and figure $5 \mathrm{a}$ are presented in figure $4 \mathrm{~b}$ and figure $5 \mathrm{~b}$, respectively. The results are rather good. We emphasize that there is no adjusting of any graytone parameter in these segmentations. This is to be contrasted with standard methods of first filtering out the noise and then performing the segmentation, which do not work well in our case.

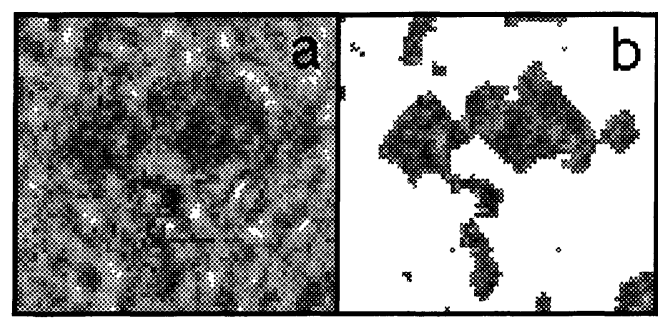

Fig. 4. - Segmentation using the automated method of a weak contrast image containing a few particles with $L_{I 1}=5, L_{I 2}=11, L_{J 2}=41$, a) portion of transmission electron micrograph containing a few metal particles [1] $\left(L_{x}=62, L_{y}=67\right)$, b) segmented image.

The same automated method has been used to segment the large transmission electron micrograph shown in figure 6. The figure contains many more particles than figures $1-5$, and the contrast varies from particles to particle. The segmentation results (Fig. 6b) show that the automated method extracts the particles with low contrast as well as the ones with high contrast.

In Figures 1-5 the automated method has been applied to images where the gray level difference between a particle and its surrounding background is comparable for all particles. Figure 6 shows that the method performs well even when these grey level differences are important. 


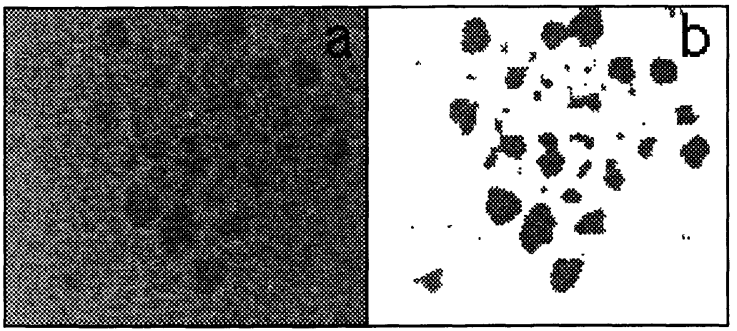

Fig. 5. - Segmentation of a weak contrast image using the automated method, with $L_{I 1}=5, L_{I 2}=$ $11, L_{J 2}=41$, a) portion of transmission electron micrograph containing several metal particles $\left(L_{x}=\right.$ $\left.132, L_{y}=150\right)$, b) segmented image.

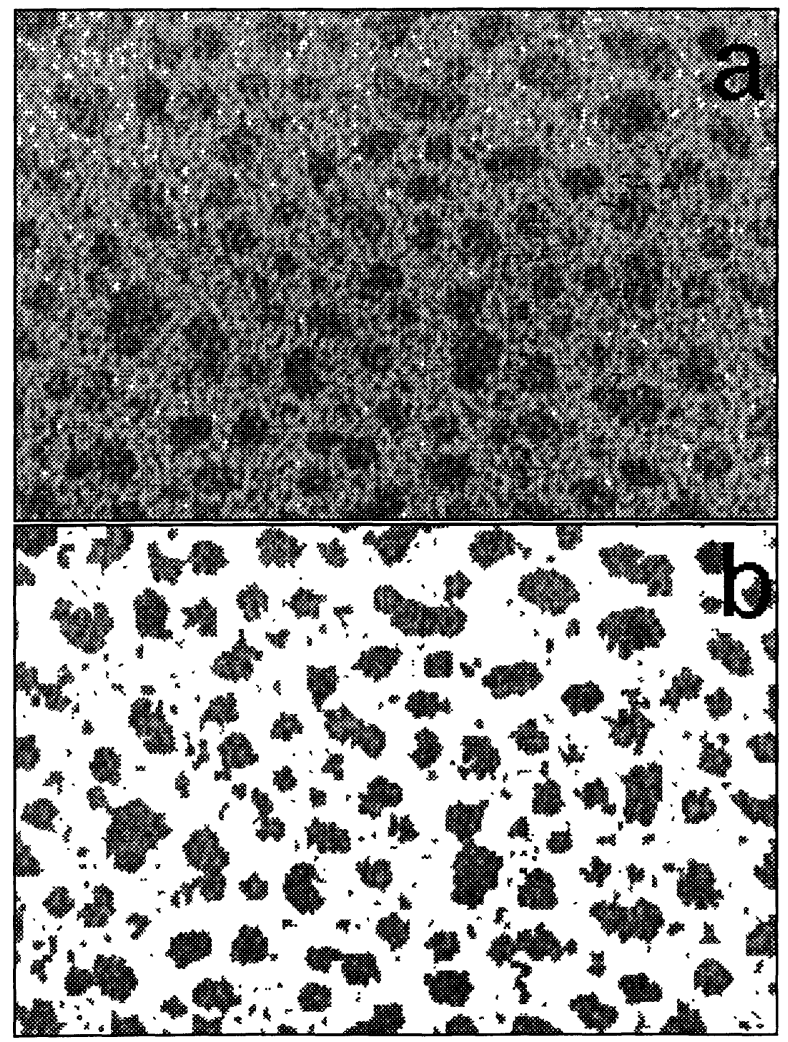

Fig. 6. - Segmentation of a transmission electron micrograph containing many metal particles [1] by the automated method with $L_{I 1}=5, L_{I 2}=11, L_{J 2}=41$, a) original image $\left(L_{x}=300, L_{y}=450\right)$, b) segmented image.

However, there are two limitations for our method: 1) The absolute gray level variation of background should not be too large. In order to overcome this difficulty, a different method has to be developed. 2) It is difficult to separate nearby particles with the method presented. This is related to the choice of the geometrical parameters, which will be discussed in the following. 


\section{Investigations of geometrical parameters.}

We now wish to study the role of the geometrical parameters. Initially, the geometrical parameters $L_{I 1}, L_{I 2}$ and $L_{J 2}$ were chosen empirically. The geometrical parameters are, in principle, related to the object size. In order to compare the different choices, we use a typical image which contains objects (particles) with very different sizes. We apply the automated method to segment the image, but change the values of the geometrical parameters. We will study the role of each one of the three parameters, keeping the other two fixed.

For a given image, different choices of the parameters can give rise to different segmentations. From the quality of the resulting segmentations, we will judge the performance of the corresponding parameter choice. As there are no objective standards for the quality of a segmentation for natural graytone images, and furthermore as the shapes of the particles in our experimental images are very irregular, we use artificial images to test the influence of geometrical parameters. Figure $7 \mathrm{a}$ is an image with two gray levels, with a white background and grey particles. The diameters of the particles vary from 1 to 31 pixels. In figure $7 \mathrm{~b}$, these particles are placed in a noisy environment and in a non-uniform background. So the figure $7 \mathrm{~b}$ is representative for the type of images we are interested in, and figure $7 \mathrm{a}$ is the expected segmentation. The segmentations of the image figure $7 \mathrm{~b}$ will be compared with figure $7 \mathrm{a}$.

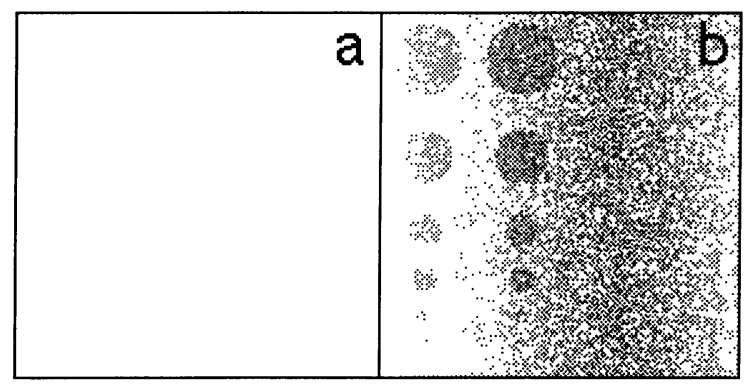

Fig. 7. - Synthetic image used to discuss the role of the geometric parameter $\left(L_{x}=150, L_{y}=150\right)$, a) original particles of varying sizes, $b$ ) the particles in a noise environment and in a non-uniform background.

4.1 VARIATION OF $L_{I 1}$. - We choose $L_{I 2}$ and $L_{J 2}$ both equal to the maximum particles size $\left(L_{I 2}=L_{J 2}=31\right.$ in Fig. 7), and set $L_{I 1}$ equal to $1,5,7,9,11$ and 15 , respectively. The resulting segmentations are shown in figure 8.

When $L_{I 1}$ is very small, the particle cannot be extracted correctly due to noise. The segmentation is simply the noise of high gray levels. When $L_{I 1}$ is increased, the noise in the segmented image decreases as shown in figure $8 \mathrm{~b}$. Thus $L_{I 1}$ sets the scale on which noise is eliminated. When $L_{I 1}$ is very large, the segmented objects are distorted in comparison with the original objects. Furthermore, the small particles are no longer detected (see Fig. 8f). In order to be able to detect the smallest particle, the value of $L_{I 1}$ must be less than the minimum particle size, $L_{\min }$. On the other hand, in order to reduce noise, the value of $L_{I 1}$ must be larger than the maximum noise scale, $L_{\text {noise }}$.

In experiments $L_{\min }$ has to be larger than $L_{\text {noise, }}$ otherwise neither our eyes nor the computer is capable of distinguishing small particles from noise. 


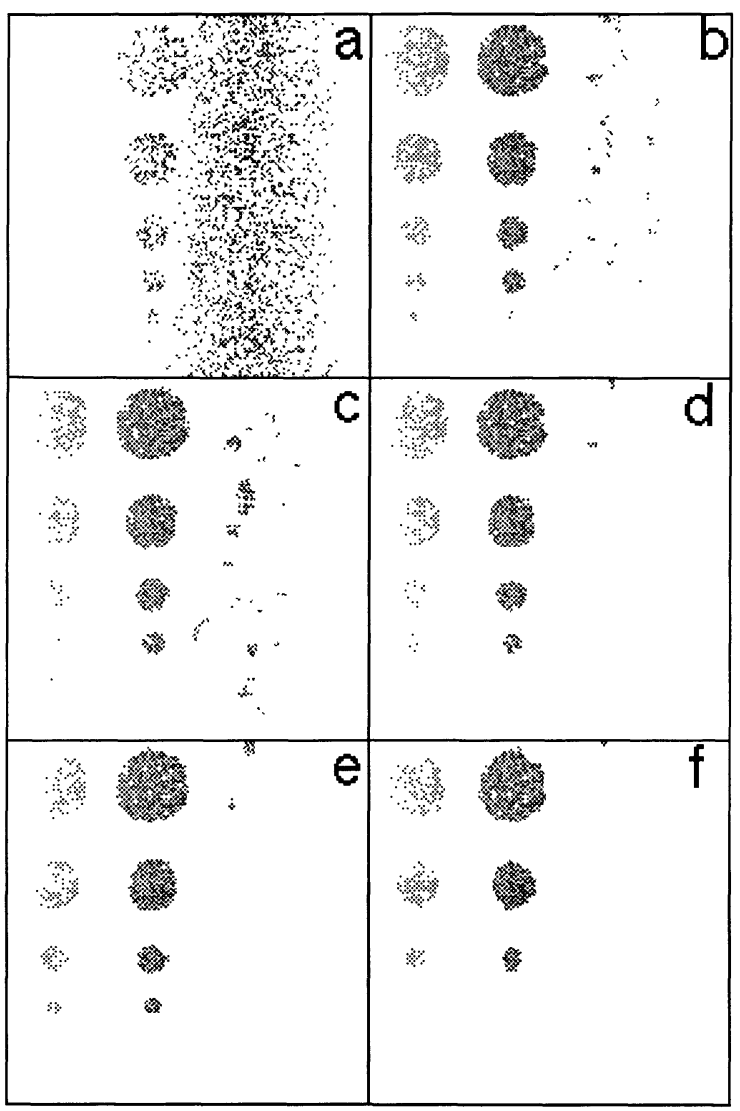

Fig. 8. - Effect of the variation of the parameter $L_{I 1}$ on the segmentation of figure $7 \mathrm{~b}$ with $L_{I 2}=L_{J 2}=$ 31: a) $L_{I 1}=1$, b) $L_{I 1}=5$, c) $L_{I 1}=7$, d) $L_{I 1}=9$, e) $L_{I 1}=11$, f) $L_{I 1}=15$.

4.2 VARIATION OF $L_{I 2}$. - We now fix $L_{I 1}=3$ and $L_{J 2}=31$, and take $L_{I 2}$ to be $5,7,9,11$, 15 and 31 respectively. For this choice of $L_{I 1}$, all the particles, down to the smallest one, can be detected. It is found that the results of the segmentations hardly vary with $L_{I 2}$. This can be understood easily: $L_{I 2}$ only influences the detection of background nuclei at the beginning of our algorithm.

4.3 VARIATION OF $L_{J 2}$. - Now, we fix $L_{I 1}=3$ and $L_{I 2}=31$, and take $L_{J 2}$ as 7, 15, 31 and 61 respectively. The resulting segmentations are shown in figure 9. When $L_{J 2}$ is close to the maximum particle size, $L_{\max }$, the results are the best ones. When $L_{J_{2}}$ is much less than $L_{\max }$, some particles cannot be detected and the shape of the large particles is distorted. This is because the function $J$ is sensitive to noise when $L_{J 2}$ is small. Under this circumstance, it is very difficult to correctly grow the background from the nuclei. If a nucleus grows into a particle region, the particle disappears partially or entirely in the segmented image.

When $L_{J 2}$ is very much larger than $L_{\max }$, the smallest particles may get lost (see Fig. 9). The reason is as follows: The gray level of the background increases from left to right in figure $7 \mathrm{~b}$, and the gray level of the particle is less than the gray level of the background on the right. As the result of the graytone variation of the background, the function $J$ between the particle and 
a background nucleus (i.e. along the joining line) decreases as $L_{\mathrm{J} 2}$ increases. Hence the nucleus can grow into the particle when $J$ is below $M_{J}$. In conclusion, to eliminate noise, $L_{J 2}$ must be chosen as large as possible but in order to overcome the effect of the background variations, $L_{J 2}$ must be chosen as small as possible. From the above experiments, we suggest that the optimal choice of $L_{J 2}$ is close to $L_{\max }$.

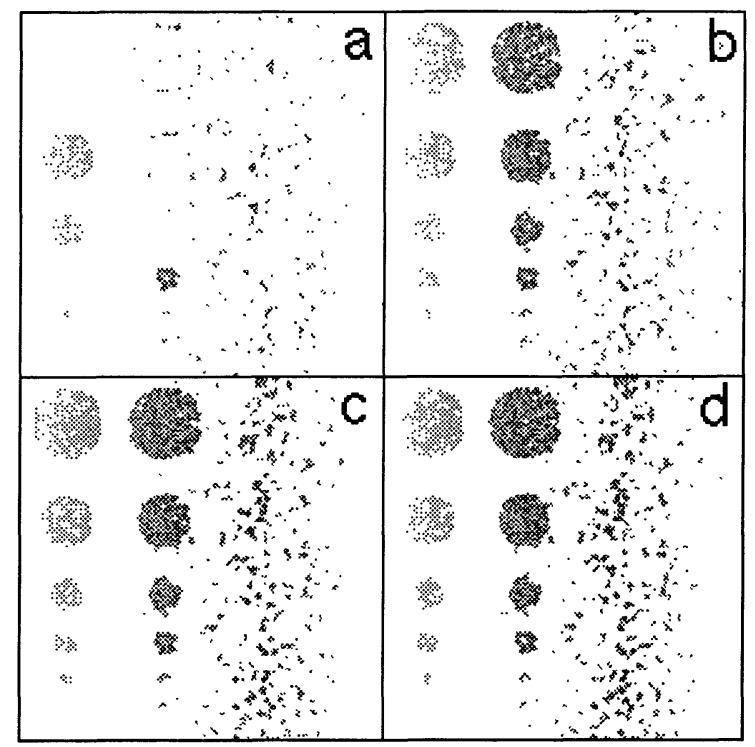

Fig. 9. - Effect of the variation of the parameter $L_{J 2}$ on the segmentation of figure $7 \mathrm{~b}$ with $L_{I 1}=3, L_{I 2}=$ 31: a) $L_{J 2}=7$, b) $L_{J 2}=15$, c) $L_{J 2}=31$, d) $L_{J 2}=61$.

\subsection{FINE STRUCTURE OF PARTICLES AND SEPARATION OF NEARBY PARTICLES.}

1) Fine structure of particles:

In the images encountered in electron microscopy, the particles (object regions) sometimes have an internal structure in the sense that there are graytone variations inside of the regions. Depending on the purpose, one either wants to enhance or suppress this internal structure.

If we want to identify the overall contours of the objects, we must suppress internal features during the segmentation operations. This can be achieved by choosing $L_{I 1}$ larger than the maximum size of the details, $L_{\text {fine }}$.

An experimental example that illustrates this point is given in the artificial image of figure 10. The fine structures is clearly visible in the particles in the original image. The corresponding gray level distribution in space is illustrated in figure $10 \mathrm{~d}$. If $L_{I 1}$ is less than $L_{\text {fine, }}$, the segmentation detects the fine structures rather than the entire particles.

2) Separation of particles:

A difficulty for segmentation algorithms is the correct separation of nearby particles. The choice of $L_{I 1}$ must take the minimum distance between particles into consideration. In addition, $L_{I 1}$ must be smaller than the smallest interparticle distance, $L_{\text {in }}$, such that nearby particles 

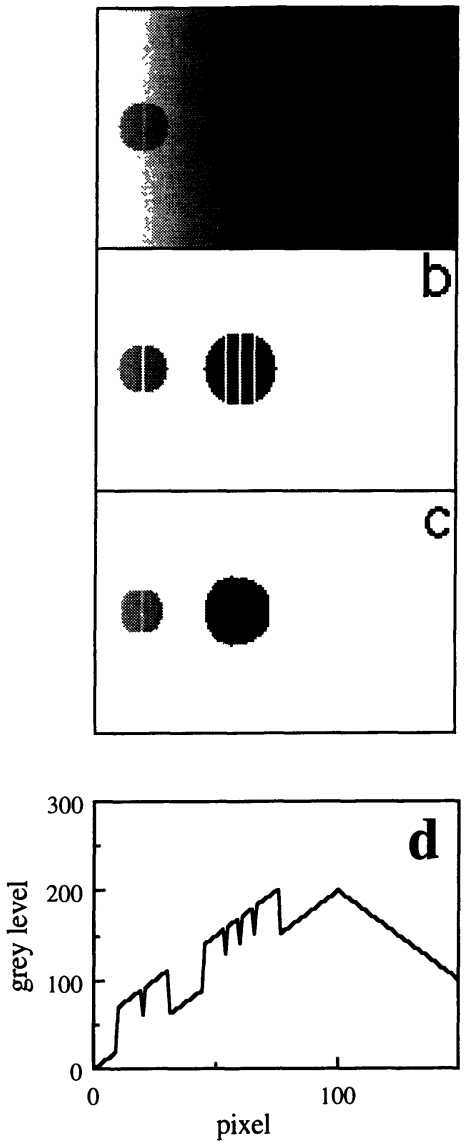

Fig. 10. - Effect of the variation of $L_{I 1}$ on the internal fine structures of the objects, for fixed $L_{I 2}=$ $L_{J 2}=31:$ a) an artificial image containing two metal particles $\left(L_{x}=100, L_{y}=150\right)$, b) segmentation with $L_{I 1}=1$, c) segmentation with $L_{I 1}=5$, d) gray level distribution of a) along the middle line from left to right.

can be separated. If $L_{I 1}$ is larger than $L_{\text {in }}$, separate, nearby particles are likely to be connected in the segmented image. This is the case in figure 11.

In figure 11, there are two particles which are very close to each other. When $L_{I 1}=3$, the two particles cannot be separated in the segmented image, but with $L_{I 1}=1$, the two particles can be separated.

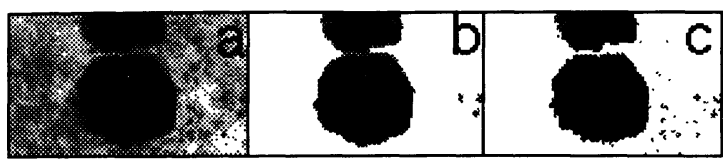

Fig. 11. - Separation of nearby particles by varying $L_{I 1}$, with fixed $L_{I 2}=L_{J 2}=41$ : a) a small portion of transmission electron micrograph containing two nearby metal particles $\left(L_{x}=60, L_{y}=99\right)$, b) segmentation $L_{I 1}=3$, c) segmentation $L_{I 1}=1$. 
In summary, the geometric parameter must be chosen on the basis of general geometrical image characteristics. $L_{J 2}$ corresponds to the maximum particle size, $L_{\max }$. $L_{I 2}$ doesn't influence the final segmentation results very much as indicated above, thus we also set it equal to $L_{\max }$. The choice of $L_{I 1}$ depends on four factors: it must be less than the smallest particle size $L_{\min }$ and the minimum separation distance between particles, $L_{\text {in }}$, and it has to be larger than the maximum noise size $L_{\text {noise }}$ and the maximum particle fine structure scale $L_{\text {fine }}$ :

$$
L_{\text {noise }}, L_{\text {fine }}<L_{I 1}<L_{\min }, L_{\text {in }}
$$

\section{Discussion.}

We have proposed an automated image segmentation algorithm based on the nucleation and growth of the background. Applying the method to a number of images shows that it is successful in extracting objects from an image which is degraded by noise and in which the background is not homogeneous. The objects can be irregular in size, shape, gray level and orientation. The extracted objects have boundaries that closely follow the true boundaries of the original image. Only very few unwanted regions are detected and only very few interesting regions are lost.

In the automated algorithm, a single graytone cutoff need to be chosen by hand. As to the geometrical parameters, we must set $L_{I 2}$ to the typical particle size ( $\left.L_{\max }\right)$ and $L_{I 1}$ has to satisfy the inequality (9).

In previous methods [10], the local threshold parameters were chosen separately for different regions when the contrast is not uniform through a image. There are two problems with such an approach: (1) How does one divide a whole image into smaller regions and (2) how does one choose the threshold values for each region? The choice influences the results.

The solution for the first problem is the multiresolution (pyramid) image representation [11], but it requires that the object be compact [12-14]. Our new method does not have this limitation, as it doesn't require subdivision of the image.

The problem of the choice of the graytone parameters is solved by the automatic parameter choice. A single cutoff $p_{\mathrm{c}}$ has to be chosen by hand. We have found that a single value for $p_{\mathrm{c}}$ assures good segmentation results for widely varying images even though the graytone parameters are highly sensitive to the graytone characteristics of each image.

Our algorithm uses global geometrical information to determine the geometrical parameters. Their optimal choice improves the quality of the particle extraction. Other segmentation methods, such as thresholding, only use graytone information but do not take into consideration the geometrical information.

If in an image the bounds (inequality (9)) for the choice of $L_{I 1}$ cannot be satisfied, it is difficult to choose an appropriate value for the segmentation. In our images of metallic particles, one sometimes encounters images for which $L_{\text {noise }}$ are close to $L_{\text {in. }}$ Then the inequality (9) cannot be satisfied any longer. To correctly identify the particles one has to choose $L_{I 1}>L_{\text {noise. }}$. As in this case $L_{I 1}>L_{\text {in }}$ or $L_{I 1} \cong L_{\text {in }}$, one must include shape information to separate these nearby particles.

\section{References}

[1] Tardy B., Noupa C., Leclercq C., Bertolili J.C., Hoareau A., Treilleux M., Faure J.P. and Nihoul G., "Catalytic hydrogenation of 1,3-butadiene on Pd particles evaporated on carbonaceous supports: particle size effect", J. Catal. 129 (1991) 1-11.

[2] Zheng X. and Kolb M., "A Particle Segmentation Method Based on Nucleation and Growth of the Background", J. Microsc. 173 Pt2 (1994) 165-172. 
[3] Adams R., "Two new methods for seeded or semi-interactive image processing" in Digital Image Computing: Techniques and Applications (APRS Melbowme, 1991) 459-465.

[4] Fu K.S. and Mui J.K., “A survey on image segmentation”, Pattern recognition 13 (1981) 3-16.

[5] Weszka J.S., "A survey of threshold selection Techniques", Comput. Graph. Image Process. 7 (1978) 259-265.

[6] Peli T. and Malah D., "A study of edge detection algorithms", Comput. Graph. Image Process. 20 (1982) 1-21.

[7] Zucker S.W., "Region growing: childhood and Adolescence”, Comput. Graph. Image Process. 5 (1976) 382-399.

[8] Lee S.U., Chung S.Y. and Park R.H., “A comparative performance study of several global thresholding techniques for segmentation", Comput. Vision, Graph. Image Process. 52 (1990) 171-190.

[9] Coster M. and Chermant J.L., Précis d'Analyse d'Image, CNRS, (Paris, 1985) 209-210.

[10] Chow C.K. and Kaneko T., "Boundary detection of radiographic images by a threshold method", Proc. IFIP Congress 71, Booklet TA-7 (North-Holland, Amsterdam, 1972) 130-134.

[11] Rosenfeld A., "Multiresolution image representation", in: S. Levialdi Ed., Digit. Image Anal. (Pitman, 1984) 18-28.

[12] Shneier M., "Using pyramids to define local thresholds for blob detection", IEEE Trans. Pattern Anal. Mach. Intell. 5 (1983) 345-349.

[13] Hong T.H. and Rosenfeld A., "Compact region extraction using weighted pixel linking in a pyramid", IEEE Trans. Pattern Anal. Mach. Intell. 5 (1984) 222-229.

[14] Hong T.H. and Shneier M., "Extracting compact objects using linked pyramids", IEEE Trans. Pattern Anal. Mach. Intell. 5 (1984) 229-237. 